\title{
Vazamento virtual: modelagem com solução analítica
}

\section{Virtual leak: modeling with analytical solution}

\author{
Francisco Tadeu Degasperi ${ }^{1}$
}

\section{RESUMO}

A tecnologia do vácuo desempenha um papel importante em incontáveis processos industriais e também em arranjos científicos e tecnológicos. A identificação das fontes de gases e vapores constitui o problema central na modelagem e nos cálculos para dimensionar as bombas de vácuo dos sistemas de vácuo. Para realizar análises detalhadas de sistemas de vácuo somente será possível se os throughputs (taxas de transferência de gás e vapor) das fontes de gases e vapores forem devidamente determinados. Em geral, as funções matemáticas que representam os throughputs das fontes de gases e vapores não são simples de ser construídas para os casos específicos e de interesse em estudo. A dificuldade em construir essas funções geralmente se dá devido a dois aspectos: um, devido à física envolvida da fonte gasosa, ou seja, como ela pode ser representada adequadamente levando-se em conta à física do fenômeno referente àquela particular fonte gasosa. O outro aspecto é quantificar, encontrar os parâmetros que especifiquem aquela particular fonte gasosa do processo em análise, ou seja, determinar a quantidade no tempo da intensidade da fonte gasosa. Em muitos casos tem-se somente um conhecimento parcial, e isso pode limitar o alcance da modelagem, a análise e o cálculo do sistema de vácuo. Dentre as possíveis fontes de gases existentes nos sistemas de vácuo, o vazamento virtual é aquela da qual se tem menos informações. Muitos textos sobre tecnologia do vácuo simplesmente o mencionam, dizendo como é prejudicial, e sugerem algumas práticas construtivas para evitar sua existência. A proposição de medidas preventivas está correta. Somente essas irão evitar o vazamento virtual. O fato de não se poder localizar o vazamento virtual, mesmo que se consiga identificá-lo, torna-o ainda mais indesejado. Ele pode comprometer seriamente o bom desempenho de um sistema de vácuo e até mesmo condená-lo definitivamente. O motivo ficará claro quando se construir seu modelo. Pretende-se, neste trabalho, estudar o vazamento virtual e construir um modelo matemático cuja solução do problema seja analítica, além de discutir como essa solução pode ser utilizada para identificar a existência de vazamento virtual nos sistemas de vácuo. Devido ao fato de construir um modelo do vazamento virtual com solução analítica, é de se esperar que simplificações sejam necessárias; não obstante, a solução obtida alcança o comportamento básico do efeito em estudo.

Palavras-chave: Vazamento virtual, Sistema de vácuo, Modelagem.

\begin{abstract}
Vacuum technology plays an important role in countless industrial processes and also in scientific and technological arrangements. The identification of the sources of gases and vapors is a central problem in the modeling and calculations for dimensioning the vacuum pumps of the vacuum systems. Detailed analysis of vacuum systems is only possible if the throughputs (gas and vapor transfer rates) of the sources of gases and vapors are properly determined. In general, the mathematical functions that represent the throughputs of the sources of gases and vapors are not simple to be constructed for the specific cases and of interest in study. The difficulty in constructing these functions is generally due to two aspects: one, due to the physics involved in the gaseous source, i.e., how adequately it can be represented by the physics of the phenomenon related to that particular gaseous source. The other aspect is to quantify, find the parameters that specify that particular gaseous source of the process being analyzed, i.e., determine the amount in time of the intensity of the gaseous source. In many cases, there is only a partial knowledge, and this may limit the scope of the vacuum system modeling, analysis, and calculation. Among the possible sources of gases in vacuum systems, virtual leakage is the one with the least information. Many texts on vacuum technology simply mention it, saying how it is harmful and suggest some constructive practices in the sense of avoiding its existence. The proposition of preventive measures is correct. In fact, only they will prevent the virtual leak! The fact that you cannot locate the virtual leak, even if you can identify it, makes it even more unwanted. It can seriously compromise the good performance of a vacuum system and even definitely condemn it. The reason will be clear when building your model. The aim of this work is to study the virtual leak and to construct a mathematical model whose solution of the problem is analytical. More, discuss how this solution can be used to identify the existence of virtual leakage in vacuum systems. Due to the fact of constructing a model of the virtual leak with analytical solution, it is expected that simplifications are necessary; nevertheless, the solution obtained reaches the basic behavior of the effect under study.
\end{abstract}

Keywords: Virtual leak, Vacuum system, Modeling.

${ }^{1}$ Centro Estadual de Educação Tecnológica Paula Souza - Faculdade de Tecnologia de São Paulo - Programa de Mestrado Profissional em Gestão e Tecnologias Produtivas - São Paulo - SP - Brasil

Autor correspondente: Francisco Tadeu Degasperi | Centro Estadual de Educação Tecnológica Paula Souza - Faculdade de Tecnologia de São Paulo Programa de Mestrado Profissional em Gestão e Tecnologias Produtivas | Email: ftd@fatecsp.br

Recebido: 13 Ago. 2018 | Aprovado: 27 Nov. 2018 


\section{INTRODUÇÃO}

Quando se trata do vazamento virtual, a seguinte questão pode ser colocada: o que é conseguido com o estudo e a construção de um modelo matemático para o vazamento virtual? Poder-se-ia simplesmente responder dizendo que há o interesse acadêmico, ou seja, o seu conhecimento puro. Mas, há também a questão prática envolvida. Com um modelo matemático do vazamento virtual, pode-se ser capaz de identificar sua presença, comparando os resultados teóricos com as medições de pressão na câmara de vácuo em função do tempo. É possível construir um modelo simples sobre ele e obter uma solução analítica de fácil aplicação e de grande alcance.

$\mathrm{Na}$ sua essência, o vazamento virtual é uma pequena quantidade de gás aprisionado em um pequeno volume - bolsão de gás - dentro da câmara de vácuo que libera seu gás para a câmara de vácuo. O vazamento virtual será prejudicial para o sistema de vácuo se a passagem que liga o pequeno volume contendo gás com a câmara de vácuo tiver condutância bastante pequena. Dessa forma, o gás aprisionado demorará muito para ser bombeado. Do ponto de vista prático, deve-se evitar o surgimento do vazamento virtual, e a regra geral é a seguinte: qualquer construção dentro do sistema de vácuo que produza volumes com pequenas passagens de gás para o vácuo deve ser evitada. Durante o processo de bombeamento em alto-vácuo, caso haja um vazamento virtual, haverá superposição dos efeitos da desgaseificação e do vazamento virtual. Isso certamente trará uma dificuldade adicional na identificação do vazamento virtual. Mais uma razão para conhecermos bem seu comportamento.

Cabe mencionar que a ideia e o conceito de vazamento virtual podem ser utilizados para identificar se peças encapsuladas estão suficientemente estanques. Em particular, as aplicações do conceito de vazamento virtual podem estar na indústria de componentes da microeletrônica - chips - e também no teste de marca-passo cardíaco.

\section{Fundamentos Teóricos e Modelagem de Sistemas de Vácuo}

\section{Processo de bombeamento em vácuo}

Antes de se apresentar qualquer resultado físico proveniente das caracterizações dos vazamentos virtuais, é necessária uma breve introdução de alguns conceitos básicos utilizados na ciência e na tecnologia do vácuo, mais informações sobre as bases teóricas do processo de bombeamento em sistemas de vácuo.

Considere-se um sistema de vácuo com várias fontes de gases e vapores possíveis presentes na câmara de vácuo. As fontes de gases e vapores possíveis são: vazamento real, vazamento virtual, vaporização, sublimação, desgaseificação, permeação, fonte gasosa da bomba de vácuo, gases e vapores de processo e injeção controlada de gases e vapores. Para cada uma dessas fontes gasosas associa-se uma quantidade de moléculas, alimentando a câmara de vácuo, variando em função do tempo. Como consequência, a ação exclusiva dessas fontes gasosas fará com que aumente a pressão na câmara de vácuo. Em contrapartida, a ação das bombas de vácuo fará com que uma quantidade de gases e vapores seja removida da câmara de vácuo num certo intervalo de tempo ${ }^{1,2}$.

Dessa forma, podem-se identificar três parcelas na equação que estabelece o balanço do número de moléculas para um intervalo de tempo na câmara de vácuo. Têm-se a parcela relativa ao número de moléculas que alimenta a câmara de vácuo devido às fontes de gases e vapores, a parcela devido à variação de pressão na câmara de vácuo ou, posto de outra forma, a variação do número de moléculas na câmara de vácuo, e, ainda, a parcela relativa ao número de moléculas removidas pela ação das bombas de vácuo. Esquematicamente, podem-se representar as três partes da equação do balanço entre a variação do número de átomos e moléculas na câmara de vácuo, conforme mostrado na Fig. 1.

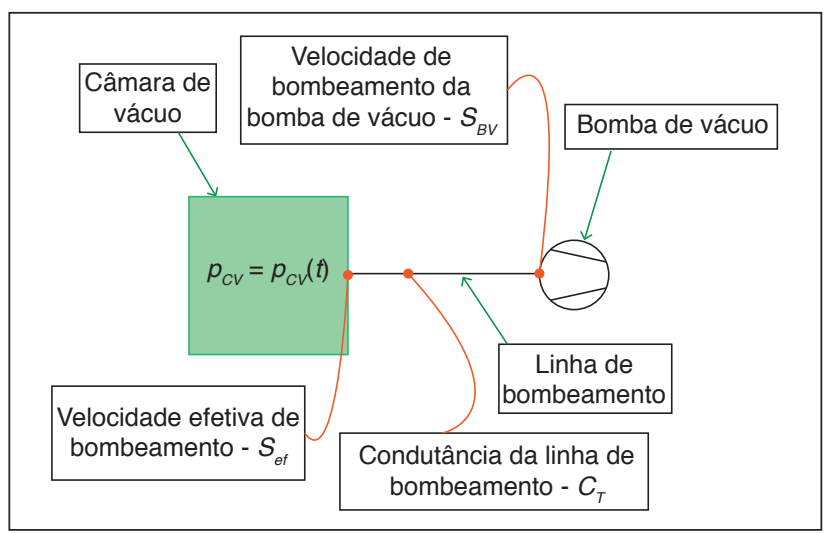

Figura 1: Configuração genérica de um sistema de vácuo com suas partes mais importantes: a câmara de vácuo, a linha de bombeamento e a bomba de vácuo.

A Fig. 1 mostra as variáveis importantes para a modelagem do sistema de vácuo. Tem-se a velocidade efetiva de bombeamento, a velocidade que efetivamente bombeia a câmara de vácuo. Essa grandeza é função tanto da condutância da linha de bombeamento como da velocidade de bombeamento da bomba de vácuo. $\mathrm{Na}$ Fig. 2 é mostrado o circuito de vácuo, explicitando a taxa de transferência de gás (throughput) em função de $S_{e \rho} S_{B V}$ e $C_{T}$. sendo que $S_{e f}$ é a velocidade efetiva de bombeamento (velocidade de bombeamento que efetivamente bombeia a câmara de vácuo), $S_{B V}$ é a velocidade de bombeamento da bomba de vácuo e $C_{T}$ é a condutância total da linha de bombeamento.

No contexto deste trabalho, é interessante determinarmos uma das principais grandezas do transporte de gases em baixas pressões, a chamada taxa de transferência de gás ou vapor [na literatura inglesa da tecnologia do vácuo, o chamado throughput (Q)]. O throughput é definido de partida como mostrado na Eq. 1:

$$
Q(t) \equiv k T \frac{d N(t)}{d t}=R T \frac{d n(t)}{d t}
$$

sendo que $k$ é a constante de Boltzmann, $T$ é a temperatura 


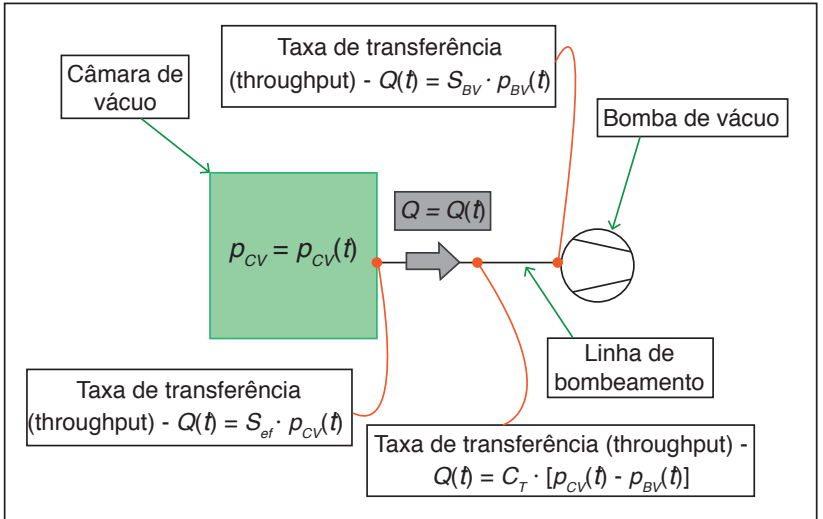

Figura 2: Configuração de um sistema de vácuo com a taxa de transferência de gás (throughput) em função das grandezas fundamentais para a modelagem de sistemas de vácuo $S_{e f}$, $S_{B V}$ e $C_{T}$. A expressão que liga essas grandezas é dada por $1 / S_{e f}=1 / S_{B V}+1 / C_{T}->S_{e f}=\left(S_{B V} C_{T}\right) /\left(S_{B V}+C_{T}\right)$.

absoluta, $N$ é o número de moléculas, $R$ é a constante universal dos gases, $n$ é o número de moles e $t$ é o tempo. O throughput é uma grandeza que depende da variação no tempo do número de moléculas em um dado recipiente (câmara de vácuo), desde que a temperatura seja conhecida. Outra forma de interpretar o throughput é dada pela Eq. 2:

$$
Q(t)=\frac{2}{3} \frac{d E(t)}{d t}
$$

ou seja, o throughput é igual a dois terços da variação no tempo de energia cinética média do movimento de translação das moléculas na câmara de vácuo. Por meio da teoria cinética dos gases, tem-se que as moléculas estão em movimento caótico incessante; assim, elas têm energia cinética ${ }^{1-4}$. A variação da pressão na câmara de vácuo leva à variação do número de moléculas de gás na câmara de vácuo; dessa forma, varia-se a energia cinética de translação da totalidade de moléculas na câmara de vácuo. Assim, a partir da Eq. 2, podemos interpretar que, durante o processo de bombeamento dos sistemas de vácuo, estamos lidando com a vazão da energia cinética média dos átomos e/ou moléculas. Observamos, também, que o throughput é dado em unidades de potência (energia na unidade de tempo) $)^{3,4}$. A Fig. 3 mostra, esquematicamente, um sistema de vácuo com as três partes principais: a câmara de vácuo (com as possíveis fontes de gases e vapores), a linha de bombeamento e a bomba de vácuo.

A Fig. 4 mostra, esquematicamente, as fontes de gases importantes que têm origem nas paredes ou superfícies internas à câmara de vácuo do sistema de vácuo ${ }^{3-6}$.

Deve ser mencionado que a dinâmica de gases em superfícies expostas ao vácuo não é um fenômeno de simples compreensão e depende de muitos fatores, entre eles a temperatura, o tipo de material da superfície e seu tratamento de limpeza e condicionamento em vácuo e, ainda, o tipo de gás.

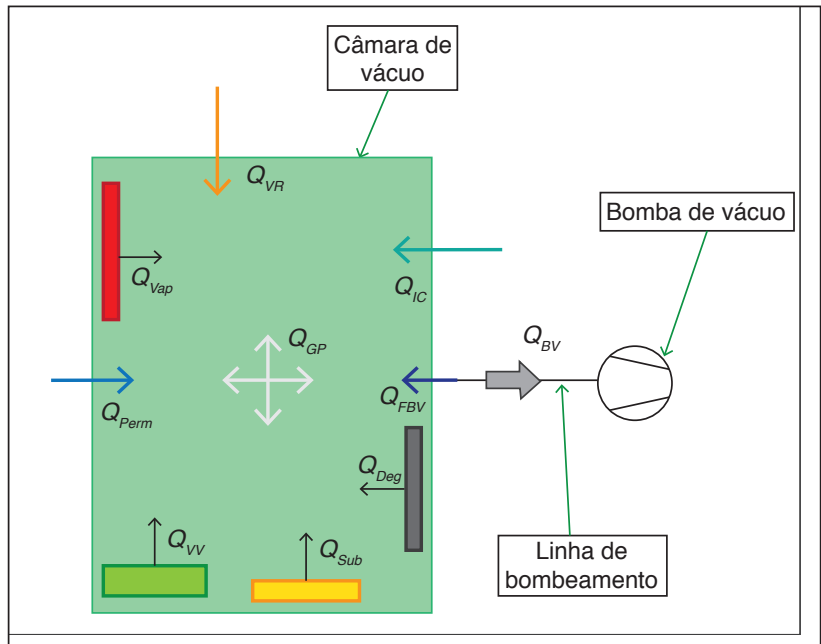

Figura 3: Sistema de vácuo mostrando as possíveis fontes de gases e vapores que alimentam a câmara de vácuo com a linha de bombeamento que, ao longo dela, os gases e vapores escoam até atingirem a bomba de vácuo. A nomenclatura das fontes de gases estará mostrada a seguir.

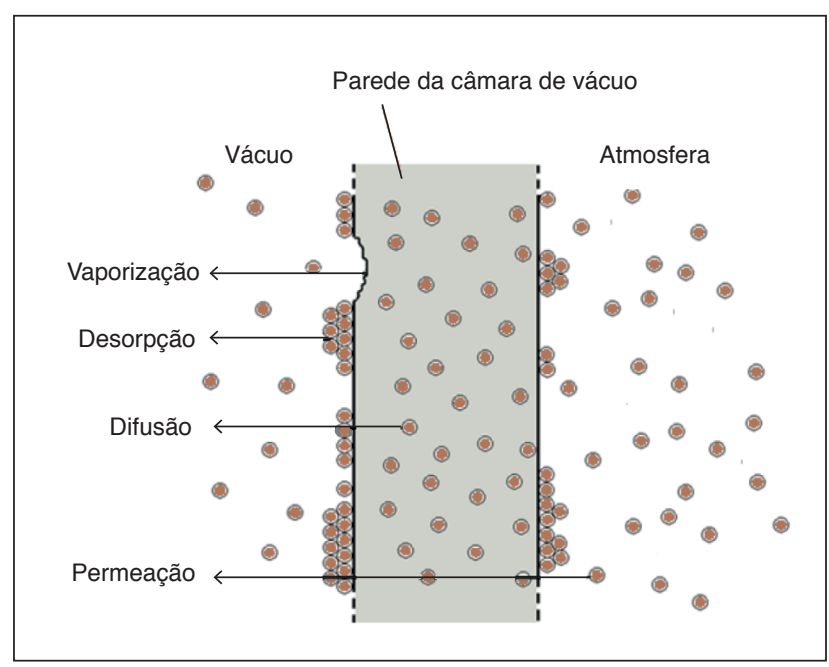

Figura 4: Configuração genérica das fontes de gases de um sistema de vácuo com origem nas superfícies expostas ao vácuo (adaptado de Harris N, Modern Vacuum Practice, 3a ed.).

Do ponto de vista do balanço do número de moléculas no sistema de vácuo, a Fig. 5 mostra, esquematicamente, as três parcelas que compõem a equação diferencial do processo de bombeamento de sistemas de vácuo. A equação utilizada na modelagem de sistemas de vácuo é uma equação diferencial ordinária no tempo. Dessa forma, a evolução da pressão no tempo é considerada na câmara de vácuo como não dependente da posição. Mesmo com essa simplificação, muitos tipos de sistemas de vácuo podem ser seguramente modelados com muito realismo. Em seguida, será desenvolvida a teoria referente ao processo de bombeamento em sistemas de vácuo.

Complementando, cabe notar que, em nosso idioma, o conceito de throughput é traduzido muitas vezes como vazão ou, ainda, fluxo. Segundo o autor deste trabalho, esses dois últimos termos são inadequados, e mesmo errados. Uma tradução direta da 


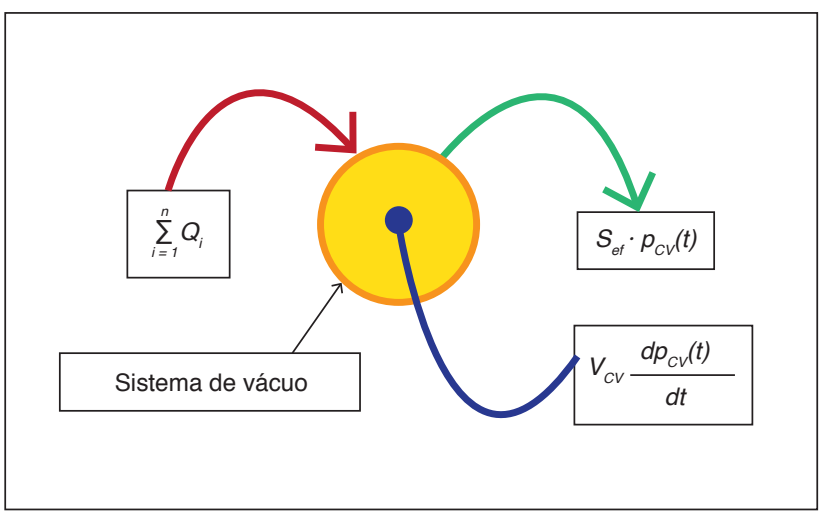

Figura 5: Sistema de vácuo mostrando as parcelas possíveis referentes ao throughput das fontes de gases e vapores que alimentam de gás a câmara de vácuo, o throughput devido ao bombeamento da bomba de vácuo e a variação de throughput na câmara de vácuo.

palavra throughput é taxa de transferência, palavra que traduz adequadamente o conceito, uma vez que vazão, na mecânica dos fluidos, significa quantidade de massa de fluido na unidade tempo que cruza uma superfície (vazão mássica). Ainda, significa o volume de fluido na unidade de tempo que cruza uma superfície (vazão volumétrica). No caso do fluido líquido, como a densidade é praticamente constante (fluido incompressível), as duas definições não trazem confusão. Ocorre que com gases - como são fluidos compressíveis - tem-se que especificar (com uma ou outra definição) de forma que a densidade do gás seja necessariamente explicitada. Dessa forma, utiliza-se, neste trabalho, o termo throughput, ou sua tradução, taxa de transferência.

Devido ao movimento de translação das moléculas, a pressão em função do tempo em sistemas de baixa pressão pode ser modelada como um processo de balanço de energia cinética dos átomos e moléculas presentes no sistema de vácuo. Assim, podemos escrever a evolução temporal da pressão no sistema de vácuo multiplicada pelo volume da câmara de vácuo como sendo igual à somatória de todas as fontes de gases presentes no sistema menos o throughput que sai do sistema. A Eq. 3 apresenta uma abordagem mais comum no cálculo do throughput para sistemas de vácuo ${ }^{4-5}$ :

$$
\begin{aligned}
V_{C V} \frac{d p_{C V}(t)}{d t} & =Q_{V R}+Q_{V V}+Q_{V a p}+Q_{S u b}+Q_{\text {Deg }} \\
& +Q_{P e r m}+Q_{F B V}+Q_{G P}+Q_{I C}-k T \frac{d N_{B V}(t)}{d t} \\
& \Rightarrow V_{C V} \frac{d p_{C V}(t)}{d t}=-k T \frac{d N_{B V}(t)}{d t}+\sum_{i=1}^{n} Q_{i}
\end{aligned}
$$

sendo $Q_{V R}$ o throughput devido ao vazamento real, $Q_{V V} \mathrm{o}$ throughput devido ao vazamento virtual, $Q_{V a p}$ o throughput devido à vaporização, $Q_{S u b}$ o throughput devido à sublimação, $Q_{D e g} \mathrm{O}$ throughput devido à desgaseificação, $Q_{\text {Perm }}$ o throughput devido à permeação, $Q_{F B V}$ o throughput devido à fonte gasosa da bomba de vácuo, $Q_{G P}$ o throughput devido aos gases e vapores de processo e $Q_{I C}$ o throughput devido à injeção controlada de gases e vapores.
A Eq. 4 apresenta a forma da equação diferencial ordinária de primeira ordem do processo de bombeamento de sistemas de vácuo com a variável dependente - a ser obtida -, que é a pressão na câmara de vácuo. Esta também apresentada à pressão inicial na câmara de vácuo, isto é, a pressão no instante do início da modelagem $^{4-5}$.

$$
\begin{aligned}
& V_{C V} \cdot \frac{d p_{C V}(t)}{d t}=-S_{e f} \cdot p_{C V}(t)+\sum_{i=1}^{n} Q_{i} \\
& V_{C V} \cdot \frac{d p_{C V}(t)}{d t}=-\frac{S_{b v} \cdot C_{\text {Total }}}{S_{b v}+C_{\text {Total }}} \cdot p_{C V}(t)+\sum_{i=1}^{n} Q_{i} \\
& p_{C V}(t=0)=p_{0}
\end{aligned}
$$

\section{Modelagem do Vazamento Virtual - Solução Analítica}

A configuração básica do vazamento virtual pode ser vista, esquematicamente, na Fig. 6. Vemos a câmara de vácuo, de volume $V_{V C^{\prime}}$, conectada a uma bomba de vácuo por meio de uma tubulação; a velocidade efetiva de bombeamento é $S_{e f}$ A câmara de vácuo - que denominaremos de câmara de vácuo principal está conectada a uma pequena câmara - que denominaremos de câmara de vácuo do vazamento virtual - de volume $V_{V V}$, por meio de uma passagem de condutância $C_{V V}$.

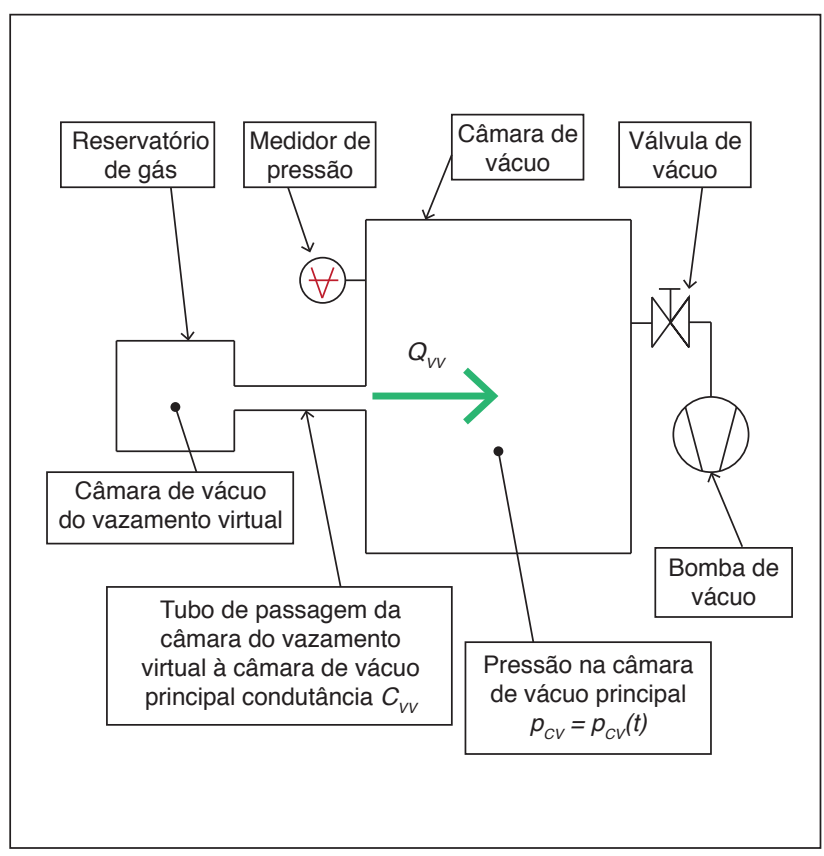

Figura 6: Sistema de vácuo mostrando a câmara de vácuo principal, a câmara de vácuo do vazamento virtual, a condutância $C_{v v}$ e a bomba de vácuo.

A denominação de vazamento virtual pode trazer alguma confusão. Precisamos ter presente que o vazamento virtual é uma de fonte de gás com características e comportamentos próprios. Seu nome tem origem no fato de o vazamento virtual poder ser confundido, na prática, com o vazamento real. Mas devemos fazer a distinção entre eles. Assim, o vazamento real é formado por uma passagem de gás entre o meio externo - meio 
da atmosfera - e o meio interno - do sistema de vácuo. Nesse caso, temos uma passagem de gás com throughput praticamente constante alimentando o sistema de vácuo. Porém, no caso do vazamento virtual, a medida que o gás contido no seu pequeno volume está sendo liberado à câmara de vácuo, sua quantidade de gás diminui até ser completamente consumida - isso se passar um tempo suficientemente grande. Fisicamente falando, a fonte de gás do vazamento real é constituída de um volume praticamente infinito - a atmosfera é o reservatório de gás. Isso faz com que a modelagem do vazamento real seja mais simples, e podemos adotar, para seu modelo, aquele estudado na injeção controlada de gás. O escoamento do gás através da passagem no vazamento real se dá em regime viscoso e, para todos os efeitos, consideramos a pressão da câmara de vácuo como sendo 0 , uma vez que essa pressão geralmente é muito menor que a pressão atmosférica. Como consequência prática, temos que o throughput do vazamento real é constante, desde que a pressão na câmara de vácuo principal seja menor que a metade da pressão atmosférica - pressão externa. Esse assunto é tratado no asssunto referente à blocagem de gás ${ }^{4,5}$.

A Fig. 7 mostra as partes essenciais componentes do vazamento virtual para a construção de sua modelagem. A imposição da sua geometria determinará quase naturalmente a dinâmica do gás nas duas câmaras de vácuo - a do vazamento virtual e a principal.

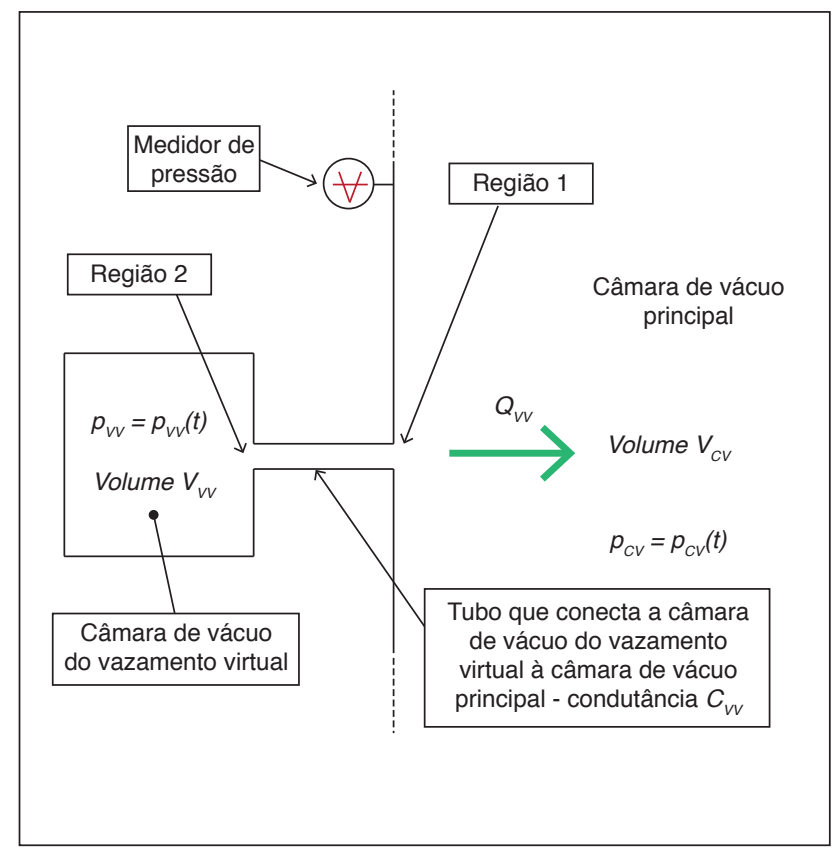

Figura 7: Câmara de vácuo do vazamento virtual - de volume $V_{v V}$ - e a câmara de vácuo principal - de volume Vcv - interligadas por uma conexão de condutância $C_{v}$.

A dinâmica do fluxo dos gases tanto na câmara de vácuo principal como na câmara de vácuo do vazamento virtual pode ser assim descrita. Consideremos que, antes de iniciar a remoção dos gases, o sistema de vácuo estava à pressão atmosférica, de modo que a pressão era a mesma em todas as suas partes
- a câmara de vácuo principal, o tubo e a câmara de vácuo do vazamento virtual. Ao iniciarmos o bombeamento dos gases, a pressão diminuirá na câmara de vácuo principal. Dessa forma, haverá diferença de pressão entre a câmara de vácuo principal e a câmara de vácuo do vazamento virtual. Como consequência, teremos o surgimento de um fluxo de gás da pressão maior - na câmara de vácuo do vazamento virtual, que é $p_{V V}=p_{V V}(t)-$, para a pressão menor - câmara de vácuo principal, que é $p_{C V}=p_{C V}(t)$. Dessa forma, os gases da câmara de vácuo do vazamento virtual estarão sendo também bombeados. Sem nenhum cálculo prévio, apenas apoiados no comportamento geral dos sistemas de vácuo, podemos dizer que acreditamos que o tempo de bombeamento dos gases na câmara do vazamento virtual dependerá da razão entre a condutância $C_{V V}$ do tubo e do volume $V_{V V}$ do vazamento virtual. Como veremos, esse resultado se confirmará.

A velocidade de bombeamento na região 2, na câmara vácuo do vazamento virtual, bem junto a uma extremidade do tubo, será chamada de $S_{2}$, e a velocidade de bombeamento na região 1, na câmara de vácuo principal, bem na outra extremidade do tubo, será chamada de $S_{1}$. Podemos relacionar essas velocidades de bombeamento e a condutância do tubo $C_{V V}$ por meio da Eq. 5 .

$$
\frac{1}{S_{2}}=\frac{1}{S_{1}}+\frac{1}{C_{V V}} \Rightarrow S_{2}=\frac{S_{1} C_{V V}}{S_{1}+C_{V V}}
$$

Para a existência "de fato" do vazamento virtual, devemos ter $C_{V V} \ll S_{1}$; assim, podemos simplificar a expressão e ficar com $S_{2} \cong C_{V V}$. Veja que, se $C_{V V}{ }^{<} S_{1}$ teremos rapidamente o gás sendo bombeado da câmara de vácuo do vazamento virtual; dessa forma, a questão referente ao vazamento na prática nem se coloca. Isso mostra que a remoção dos gases da câmara de vácuo do vazamento virtual é determinada pela condutância do tubo que liga a câmara de vácuo principal com a câmara de vácuo do vazamento virtual. Assim, apesar de a velocidade de bombeamento da bomba de vácuo $S_{b}$ poder ser de alto valor, o que determinará a queda da pressão na câmara de vácuo do vazamento virtual será a razão $C_{V V} / V_{V V^{*}}$. Nesse sentido, devemos esperar, na região de alto-vácuo, três etapas bem distintas na curva da pressão em função do tempo na câmara de vácuo principal. Na primeira etapa, teremos a queda de pressão devido à remoção dos gases remanescentes do prévácuo. Na segunda, a pressão cai lentamente devido aos gases que saem da câmara de vácuo do vazamento virtual. Finalmente, na terceira etapa, a pressão na câmara de vácuo principal atinge seu valor mínimo, ou seja, a pressão final $-p_{\text {final }}$.

Em cada uma das etapas apresentadas, referentes ao processo de bombeamento da câmara de vácuo principal, as fontes de gases presentes no sistema de vácuo participam conjuntamente. Em geral, teremos a predominância de uma particular fonte de gás durante uma etapa do processo de bombeamento. Ainda, se uma determinada fonte de gás for demasiadamente intensa digamos a sublimação de um determinado material -, poderá ocorrer que a fonte de gás relativa ao vazamento virtual seja 
completamente irrelevante frente àquela. Assim, devemos analisar cada caso e cada etapa do sistema de vácuo de forma a considerar as particularidades e os aspectos marcantes do processo de bombeamento em questão. Uma análise detalhada em sistemas de vácuo somente poderá ser realizada se a identificação e a quantificação das fontes de gases e vapores forem feitas. Essa é a tarefa mais importante e difícil na realização da modelagem de sistemas de vácuo.

Continuando, vamos escrever a equação diferencial fundamental para o processo de bombeamento em vácuo - $E_{P B V}$ - para a câmara de vácuo principal e também para a câmara de vácuo do vazamento virtual. A modelagem de sistemas de vácuo utilizando a equação diferencial mostrada a seguir é estudada em detalhe nos apêndices $\mathrm{A}$ e $\mathrm{D}^{4,5}$. No caso da câmara de vácuo principal, temos a equação $E_{P B V}$ mostrada na Eq. 6:

$$
V_{C V} \frac{d p_{C V}(t)}{d t}=-S_{e f C V} \cdot p_{C V}(t)+\sum_{i=1}^{n} Q_{i}
$$

sendo que a parcela $\sum_{i=1}^{n} Q_{i}$ é a totalidade das fontes de gases presentes no sistema de vácuo. Consideraremos, no caso em estudo, apenas a presença do vazamento virtual e da desgaseificação. Adotaremos para a fonte de gás, devido à desgaseificação, simplesmente um valor constante; isso deve ser imposto como uma das condições para que a pressão final do sistema de vácuo atinja um valor constante $-p_{\text {final }}$ Escrevemos matematicamente a presença das duas fontes de gás como mostrado na Eq. 7.

$$
\sum_{i=1}^{n} Q_{i}=Q_{V V}+Q_{D e g}
$$

Seguindo em nossa análise, vamos introduzir essa última função, a expressão Eq. 7, na equação diferencial $-E_{P B V}$ - para a câmara de vácuo principal, obtendo a Eq. 8:

$$
\begin{aligned}
& V_{C V} \frac{d p_{C V}(t)}{d t}=-S_{e f C V} \cdot p_{C V}(t)+\sum_{i=1}^{n} Q_{i} \Rightarrow \\
& V_{C V} \frac{d p_{C V}(t)}{d t}=-S_{e f C V} \cdot p_{C V}(t)+Q_{V V}+Q_{D e g}
\end{aligned}
$$

Prosseguindo, podemos expressar o throughput devido à desgaseificação, em termos da pressão final, da seguinte forma: $Q_{D e g}=S_{e f C V} \times p_{\text {final }}$ com $S_{\text {efCV }}$ à velocidade efetiva de bombeamento na câmara de vácuo principal. Ainda, o throughput de origem no vazamento virtual pode ser escrito como $Q_{V V}(t)=C_{V V}\left[p_{v v}(t)-p_{c v}(t)\right]$. Dessa maneira, temos que a equação $E_{P B V}$ toma a seguinte forma mostrada na Eq. $9^{7-8}$.

$$
\begin{aligned}
V_{C V} \frac{d p_{C V}(t)}{d t} & =-S_{e f C V} \cdot p_{C V}(t)+1 \\
& +C_{V V}\left[p_{V V}(t)-p_{C V}(t)\right]+S_{e f C V} \cdot p_{\text {final }}
\end{aligned}
$$

Reagrupando os termos da expressão Eq. 9, ficamos com uma forma adequada para a resolução dessa equação diferencial. Explicitando, a equação diferencial do processo de bombeamento de gases da câmara de vácuo principal fica conforme a Eq. 10 .

$$
\begin{aligned}
V_{C V} \frac{d p_{C V}(t)}{d t} & =-S_{e f C V}\left[p_{C V}(t)-p_{f i n a l}\right]+ \\
& +C_{V V}\left[p_{V V}(t)-p_{C V}(t)\right]
\end{aligned}
$$

Vemos que, para resolver a Eq.10, precisamos conhecer a função $p_{v v}=p_{v v}(t)$, ou seja, precisamos saber como varia no tempo a pressão na câmara de vácuo do vazamento virtual. Continuando com a análise, vamos escrever a equação diferencial fundamental para o processo de bombeamento em vácuo - $E_{P B V}$ - na câmara de vácuo do vazamento virtual; temos, assim, mostrada na Eq. 11:

$$
\begin{aligned}
& V_{V V} \frac{d p_{V V}(t)}{d t}=-S_{e f V V} \cdot p_{V V}(t)+\sum_{i=1}^{n} Q_{i} \Rightarrow \\
& V_{V V} \frac{d p_{V V}(t)}{d t}=-\frac{S_{1} \cdot C_{V V}}{S_{1}+C_{V V}} \cdot p_{V V}(t)+\sum_{i=1}^{n} Q_{i}
\end{aligned}
$$

sendo que $S_{e f V V}$ é a velocidade efetiva de bombeamento na câmara de vácuo do vazamento virtual, que pode ser escrita como $S_{e f V V}=\left(S_{1} \times C_{v v}\right) /\left(S_{1}+C_{v v}\right)$, e a parcela representada pela somatória é a totalidade das fontes de gases presentes na câmara de vácuo do vazamento virtual. Consideraremos, no caso do vazamento virtual, somente a fonte de gás devida à desgaseificação. Adotaremos, simplesmente, uma desgaseificação constante que fará com que, nessa câmara de vácuo, a pressão final atinja o valor $p_{\text {final }}$. Assim, podemos escrever:

$$
\sum_{i=1}^{n} Q_{i}=Q_{\text {Deg }}
$$

e o processo de bombeamento na câmara de vácuo do vazamento virtual ficará expresso como mostrado na equação diferencial $E_{P B V}-$ mostrada na Eq. 12.

$$
\begin{gathered}
V_{V V} \frac{d p_{V V}(t)}{d t}=-S_{e f V V} \cdot p_{V V}(t)+\sum_{i=1}^{n} Q_{i} \Rightarrow \\
V_{V V} \frac{d p_{V V}(t)}{d t}=-S_{e f V V} \cdot p_{V V}(t)+Q_{D e g}
\end{gathered}
$$

Continuando, mais uma vez podemos expressar o throughput devido à desgaseificação, em termos da pressão final na câmara de vácuo do vazamento virtual, da seguinte forma: $Q_{\text {Deg }}=S_{e f V V} p_{\text {final }}$. Explicitamente, estamos adotando que a pressão final na câmara de vácuo principal é a mesma que na câmara de vácuo do vazamento virtual. Essa é uma suposição bastante razoável e nada restritiva, não alterando a essência da modelagem do vazamento virtual. Assim, a expressão para o processo de 
bombeamento na câmara de vácuo do vazamento virtual é dada pela equação - $E_{P B V}$ - mostrada na Eq. 13.

$$
\begin{gathered}
V_{V V} \frac{d p_{V V}(t)}{d t}=-S_{e f V V} \cdot p_{V V}(t)+S_{e f V V} \cdot p_{\text {final }} \Rightarrow \\
V_{V V} \frac{d p_{V V}(t)}{d t}=-S_{e f V V}\left[p_{V V}(t)-p_{\text {final }}\right]
\end{gathered}
$$

A velocidade efetiva de bombeamento na câmara de vácuo do vazamento virtual $S_{e f V V}$ é identificada com $S_{2}$. Dessa forma, temos que $S_{e f V V}=S_{2}$. A solução da equação $-E_{P B V}$ para a modelagem da câmara de vácuo do vazamento virtual - é dada pela função mostrada na Eq. 13.

$$
p_{V V}(t)=\left(p_{V V}^{0}-p_{\text {final }}\right) \exp \left(-\frac{S_{e f V V}}{V_{V V}} t\right)+p_{\text {final }}
$$

Foi imposta que a condição inicial do problema é dada por $p_{V V}(t=0)=p_{v v}^{0}$. Dessa forma, a função $p_{V V}=p_{V V}(t)$ representa matematicamente a variação temporal da pressão na câmara de vácuo do vazamento virtual. De posse dessa última função, podemos considerar novamente a equação $E_{P B V}$ escrita para a câmara de vácuo principal. Dessa maneira, reescrevendo-a, temos a Eq. 14.

$$
\begin{aligned}
V_{C V} \frac{d p_{C V}(t)}{d t} & =-S_{e f C V}\left[p_{C V}(t)-p_{f i n a l}\right]+ \\
& +C_{V V}\left[p_{V V}(t)-p_{C V}(t)\right]
\end{aligned}
$$

A partir dessa última equação, e nela introduzindo, explicitamente, a função $p_{V V}=p_{V V}(t)$, ficamos com a seguinte equação $-E_{P B V}$ para a câmara de vácuo principal - mostrada na Eq. 15.

$$
\begin{aligned}
V_{C V} \frac{d p_{C V}(t)}{d t} & =-\left(S_{e f C V}+C_{V V}\right) p_{C V}(t)+ \\
& +C_{V V}\left(p_{V V}^{0}-p_{\text {final }}\right) \exp \left(-\frac{S_{e f V V}}{V_{V V}} t\right)+\left(C_{V V}+S_{e f C V}\right) p_{\text {final }}
\end{aligned}
$$

Reagrupando seus termos, finalmente ficamos com a seguinte equação mostrada na Eq. 16.

$$
\begin{aligned}
\frac{d p_{C V}(t)}{d t} & =-\left(\frac{S_{e f C V}+C_{V V}}{V_{C V}}\right) p_{C V}(t)+ \\
& +\left[\frac{C_{V V}\left(p_{V V}^{0}-p_{\text {final }}\right)}{V_{C V}}\right] \exp \left(-\frac{S_{e f V V}}{V_{V V}} t\right)+\left(\frac{\left(C_{V V}+S_{e f C V}\right)}{V_{C V}}\right) p_{\text {final }}
\end{aligned}
$$

Apesar de trabalhosa, a solução dessa última equação diferencial ordinária de primeiro grau pode ser obtida sem muita dificuldade. Estaremos impondo, como condição inicial, que a pressão na câmara de vácuo principal, em $t=0$, seja igual a
$p_{C V}(t=0)=p_{C V}^{0}$. Assim, a última equação diferencial tem como solução a seguinte função mostrada na Eq. 17.

$$
\begin{aligned}
& p_{C V}(t)=\left[p_{C V}^{0}-\frac{C_{V V}\left(p_{V V}^{0}-p_{\text {final }}\right)}{\left.S_{e f C V}+C_{V V}-\frac{S_{e f V V} \cdot V_{C V}}{V_{V V}}-p_{\text {final }}\right]}\right. \\
& \exp \left[-\frac{S_{e f C V}+C_{V V}}{V_{C V}} t\right]+\left[\frac{C_{V V}\left(p_{V V}^{0}-p_{f i n a l}\right)}{S_{e f C V}+C_{V V}-\frac{S_{e f V V} \cdot V_{C V}}{V_{V V}}}\right] \\
& \exp \left(-\frac{S_{e f V V}}{V_{V V}} t\right)+p_{\text {final }}
\end{aligned}
$$

A função $p_{C V}=p_{C V}(t)$ descreve matematicamente a evolução temporal da pressão na câmara de vácuo principal. É composta de três partes, conforme adiantamos anteriormente em discussão preliminar. A primeira parcela da função $p_{C V}=p_{C V}(t)$ mostra os efeitos dominantes relativos aos gases de origem na própria câmara de vácuo principal. A segunda mostra os efeitos relativos aos gases da câmara do vazamento de vácuo do vazamento virtual na câmara de vácuo principal. Finalmente, a terceira e última parcela mostra simplesmente o efeito da desgaseificação residual do sistema de vácuo. Vemos que a importância, mais ou menos acentuada, do vazamento virtual, na evolução temporal na pressão na câmara de vácuo principal, dependerá da relação entre as várias grandezas pertinentes do sistema de vácuo. Ainda, na função $p_{C V}=p_{C V}(t)$ participam grandezas com valores bastante distintos entre si. Dessa forma, algumas simplificações podem ser conseguidas, tornando a expressão mais fácil de ser manipulada e os cálculos mais simples e adequados para tratar as situações realistas, sem alterar, certamente, seu conteúdo.

Com o propósito de manter as duas funções que representam a evolução temporal da pressão nas duas câmaras de vácuo, escreveremos $p_{V V}=p_{V V}(t)$ na Eq. 18.

$$
p_{V V}(t)=\left(p_{V V}^{0}-p_{\text {final }}\right) \exp \left(-\frac{S_{e f V V}}{V_{V V}} t\right)+p_{\text {final }}
$$

Iniciaremos observando os vários termos da função $p_{C V}=p_{C V}(t)$ e procedendo às simplificações aceitáveis após análise física. Assim, enquanto o vazamento virtual tiver importância, a pressão na câmara de vácuo do vazamento virtual será sempre muito maior que a pressão na câmara de vácuo principal. Caso isso não se verifique, o vazamento virtual deixa de ser importante e praticamente a questão sobre sua existência nem se coloque.

Continuando, existindo o vazamento virtual, podemos escrever que $p_{V V}(t)>>p_{C V}(t)$ para todos os instantes de tempo. A condutância $C_{V V}$ é o fator que limita, ou seja, é o fator determinante do processo de bombeamento dos gases da câmara de vácuo do vazamento virtual; dessa maneira, $S_{e f V V} \cong C_{V V^{*}}$. Com relação às pressões iniciais nas câmaras de vácuo, seguramente temos verificadas as seguintes desigualdades: $p_{V V}^{0}>>p_{\text {final }}$ e $p_{C V}^{0}>>p_{\text {final }}$ - essas hipóteses estavam presentes desde o início da construção do modelo do vazamento 
virtual e as vemos coerentemente explicitadas na função $p_{C V}(t)=p_{C V}(t)$.

Ainda, a velocidade efetiva de bombeamento na câmara de vácuo principal será sempre muito maior que a velocidade efetiva de bombeamento na câmara de vácuo do vazamento virtual - caso contrário, não teríamos o vazamento virtual; então, $S_{e f C V} \gg S_{e f V V} \cong C_{V V}$. Levando-se em conta essas aproximações apresentadas, podemos reescrever os termos da função $p_{C V}(t)=p_{C V}(t)$ como segue o termo simplificado:

$$
\left[p_{C V}^{0}-\frac{C_{V V}\left(p_{V V}^{0}-p_{\text {fianl }}\right)}{S_{e f C V}+C_{V V}-\frac{S_{e f V V} V_{C V}}{V_{V V}}}-p_{\text {final }}\right] \cong\left[p_{C V}^{0}-\frac{C_{V V} p_{V V}^{0}}{S_{e f C V}-\frac{C_{V V} V_{C V}}{V_{V V}}}\right]
$$

e também, para o outro termo, temos sua simplificação:

$$
\left[\frac{C_{V V}\left(p_{V V}^{0}-p_{f i n a l}\right)}{S_{e f C V}+C_{V V}-\frac{S_{e f V} V_{C V}}{V_{V V}}}\right] \cong\left[\frac{C_{V V} p_{V V}^{0}}{S_{e f C V}-\frac{C_{V V} V_{C V}}{V_{V V}}}\right]
$$

De posse dessas simplificações e introduzindo-as na expressão de $p_{C V}(t)=p_{C V}(t)$, temos a Eq.19.

$$
\begin{aligned}
p_{C V}(t) & \cong\left[p_{C V}^{0}-\frac{C_{V V} p_{V V}^{0}}{S_{e f C V}-\frac{C_{V V} V_{C V}}{V_{V V}}}\right] \exp \left(-\frac{S_{e f C V}}{V_{C V}} t\right)+ \\
& +\left[\frac{C_{V V} p_{V V}^{0}}{S_{e f C V}-\frac{C_{V V} V_{C V}}{V_{V V}}}\right] \exp \left(-\frac{C_{V V}}{V_{V V}} t\right)+p_{f i n a l}
\end{aligned}
$$

Finalmente, encontramos a expressão que representa a evolução temporal da pressão na câmara de vácuo principal. Nessa expressão estão sendo consideradas, como fontes de gases, as de origem no vazamento virtual e na desgaseificação das paredes da câmara de vácuo principal. Poderíamos, em princípio, ter considerado a desgaseificação dependente do tempo, como estudado ${ }^{4}$. Nesse caso, mesmo não havendo solução analítica para a equação diferencial para o processo de bombeamento em vácuo $-E_{P B V}-$, lançaríamos mão de métodos numéricos, tais como o de EulerHeun ou de Runge-Kutta de $4^{\mathrm{a}}$ ordem para chegarmos à solução. Mesmo não considerando o aspecto - mais realista - da evolução temporal da desgaseificação na modelagem do vazamento virtual, os resultados alcançados mostram seu comportamento essencial em um sistema de vácuo.

Podemos explorar um pouco mais a questão da desgaseificação na modelagem realizada. Sabemos que as várias fontes de gases que concorrem nos sistema de vácuo manifestam-se geralmente ao mesmo tempo. Temos que uma fonte de gás será mais ou menos importante dependendo de sua intensidade durante uma etapa específica do processo de bombeamento. Pode ocorrer, por exemplo, que a desgaseificação seja tão intensa em um sistema de vácuo que torne a fonte de gás, devido ao vazamento virtual, completamente imperceptível. Nessa situação, o vazamento virtual poderá não trazer problemas. Mas nem sempre a realidade é assim. Devido aos possíveis cenários apresentados estarem dentro do plano da realidade, deveremos ter um conhecimento suficiente das várias fontes gasosas a ponto de discerni-las e quantificá-las. Isso justifica, mais uma vez, a construção de modelos capazes de representar matematicamente as fontes de gases presentes nos sistemas de vácuo.

Voltando ao vazamento virtual, esse pode também ser prejudicial em um sistema de vácuo quando vários gases de processo são empregados. Digamos que, após o bombeamento, atingiu-se uma dada pressão na câmara de vácuo principal. Em seguida, um determinado gás é injetado na câmara de vácuo principal; esse gás preencherá parcial ou totalmente a câmara de vácuo do vazamento virtual. Posteriormente, com a interrupção da entrada do gás injetado, uma pressão mais baixa será novamente atingida. Agora, um outro gás de processo é injetado na câmara de vácuo principal. Mas o gás injetado anterior ainda estará suprindo a câmara de vácuo principal, uma vez que a fonte do vazamento virtual está presente. Isso pode ser extremamente prejudicial para o processo em andamento. Poderá ter efeito contaminante, assim, apesar de nem sempre estarmos preocupados com o valor da pressão final; o vazamento virtual será uma fonte de gás que poderá prejudicar as etapas de um processo. Esse fato é de extrema importância em vários processos de fabricação de produtos, por exemplo, circuitos integrados na indústria de microeletrônica, na fabricação de discos compactos - CD e DVD - indústria de lâmpadas de descargas em gases, fabricação de superfícies construídas por filmes finos e outros mais.

Podemos também usar os resultados obtidos neste estudo sobre o vazamento virtual para testar a estanqueidade de peças seladas. Digamos, por exemplo, que os vários invólucros, bem distintos um do outro, um marca-passo cardíaco, um circuito integrado ou, ainda, uma lata de leite condensado deverão ter a estanqueidade testada. Podemos proceder da seguinte forma: inicialmente submetemos uma dada peça a uma alta pressão - duas ou três atmosferas - de gás hélio, com duração de tempo determinada por normas de operação técnicas.

Assim, se a peça tiver um furo que determina uma passagem do meio exterior ao seu interior, mesmo que muito pequena, ou seja, pequena condutância, uma quantidade de hélio entrará nela. Em seguida, colocamos a peça em uma câmara de vácuo, acoplada a um detector de vazamentos ou a um analisador de gases residuais. Assim, poderemos monitorar a evolução temporal da pressão parcial do hélio. Pelo exposto, se a evolução temporal da pressão parcial do hélio for do tipo representada pela Eq. 20 estaremos diante de um vazamento virtual, significando que a peça tem um furo com passagem ao seu interior.

Deve ficar claro que esse tipo de procedimento requer alguns testes e calibrações para sua validação. Esses procedimentos de validação da metodologia e de sua quantificação não são muito 
difíceis, requerendo, essencialmente, uma compreensão dos conceitos básicos envolvidos.

$$
\begin{aligned}
p_{C V}(t) & \cong\left[p_{C V}^{0}-\frac{C_{V V} p_{V V}^{0}}{S_{e f C V}-\frac{C_{V V} V_{C V}}{V_{V V}}}\right] \exp \left(-\frac{S_{e f C V}}{V_{C V}} t\right)+ \\
& +\left[\frac{C_{V V} p_{V V}^{0}}{S_{e f C V}-\frac{C_{V V} V_{C V}}{V_{V V}}}\right] \exp \left(-\frac{C_{V V}}{V_{V V}} t\right)+p_{f i n a l}
\end{aligned}
$$

\section{RESULTADOS CALCULADOS}

Finalizando este estudo sobre vazamento virtual, vamos mostrar como é possível obter as grandezas que o definem, isto é, a condutância $C_{V V}$ e o volume da câmara de vácuo do vazamento virtual $V_{V V^{*}}$ Por meio da Fig. 8, vemos a evolução temporal da pressão na câmara de vácuo principal.

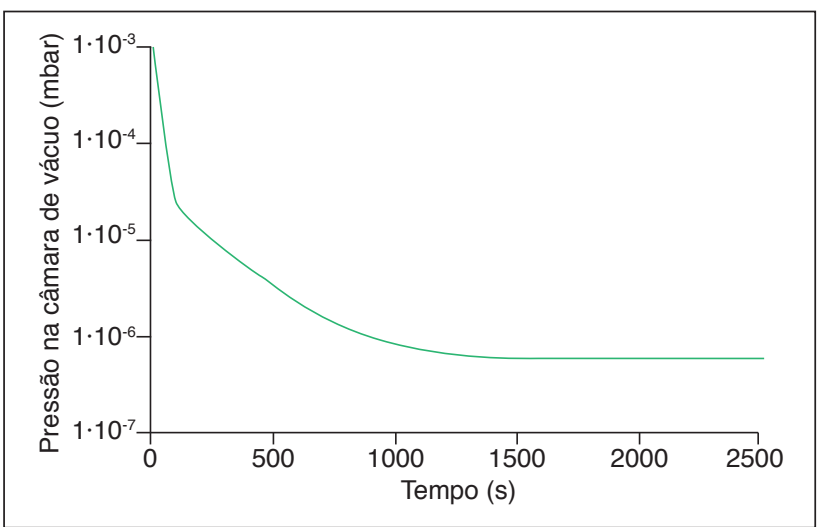

Figura 8: Função temporal da pressão na câmara de vácuo principal com a presença de um vazamento virtual.

Figura 8 tem os seguintes valores para as grandezas pertinentes na modelagem do vazamento virtual: $V_{V V}=100 \mathrm{~L}, S_{e f C V}=5 \mathrm{~L} / \mathrm{s}$, $V_{V V}=3 \times 10^{-5} \mathrm{~L}, C_{V V}=1,5 \times 10^{-7} \mathrm{~L} / \mathrm{s}, p_{C V}^{0}=1 \times 10^{-3} \mathrm{mbar}$, $p_{V V}^{0}=1000$ mbar e $p_{\text {final }}=6 \times 10^{-7}$ mbar. O primeiro trecho reto da curva refere-se à evolução temporal da pressão devido ao gás do volume. Nessa etapa do bombeamento, em geral, as outras fontes gasosas não têm importância significativa e, na maior parte dos casos, são completamente desprezíveis. Analisando o segundo trecho reto da curva da função $p_{C V}=p_{C V}(t)$, vemos que podemos extrair os valores das grandezas determinantes do vazamento virtual a partir do valor do seu coeficiente angular, que denominamos $\alpha$. Continuando, a interseção desse segundo trecho de reta, com o eixo da pressão na câmara de vácuo principal, fornece-nos o fator que multiplica a segunda exponencial da função $p_{C V}=p_{C V}(t)$, que denominamos $\beta$. Dessa forma, temos um sistema de duas equações algébricas e duas variáveis explicitadas a seguir.

Vemos que assumimos que as outras grandezas presentes nesse sistema algébrico são consideradas conhecidas.

Ilustraremos com alguns exemplos o efeito do vazamento virtual nos sistemas de vácuo. São apresentados três casos de estudo, tendo o vazamento virtual presença mais ou menos pronunciada.
No primeiro caso (Fig. 9), apesar de sua presença existir e ser notada, o vazamento virtual, em aproximadamente 300 segundos, deixa de ser uma fonte de gás importante para o sistema de vácuo. Isso não significa que ele não possa ser danoso ao processo em

$$
\left\{\begin{array}{l}
-\frac{C_{V V}}{V_{V V}}=\alpha \\
\frac{C_{V V} p_{V V}^{0}}{S_{e f C V}-\frac{C_{V V} V_{C V}}{V_{V V}}}=\beta
\end{array}\right.
$$

vácuo. Por exemplo, pode ocorrer que, nesse ínterim, haja introdução de um gás de processo e, ainda, o gás presente na câmara de vácuo do vazamento virtual estará sendo liberado. Essa mistura pode ser completamente inadmissível.

No segundo caso (Fig.10) de estudo, o gás do vazamento virtual demora em torno de 1.200 segundos para ser bombeado. Finalmente, no terceiro caso (Fig. 11), o vazamento virtual não tem participação no decorrer do processo de bombeamento dos gases e vapores da câmara de vácuo principal. A relação $C_{V V} / V_{V V}$ define a participação do vazamento virtual para a evolução temporal da pressão na câmara de vácuo principal.

Podemos ainda dizer que essa última relação define se há ou não vazamento virtual e, com isso, que não basta termos uma pequena câmara de vácuo - bolsão - com uma passagem ligando ao sistema de vácuo, matematicamente falando. Não importam os valores isolados de $C_{V V}$ e $V_{V V}$; importante para a própria definição do vazamento virtual é a relação $C_{V V} / V_{V V}$. Nos casos estudados, foram adotados valores realistas para as variáveis que aparecem na expressão $p_{C V}=p_{C V}(t)$. Poderíamos, ainda, ter adotado valores para $C_{V V}$ e $V_{V V}$, os quais tornariam a fonte de gás do vazamento virtual atuando na queda da pressão na câmara de vácuo principal por dias, semanas, meses ou, ainda, tempos maiores.

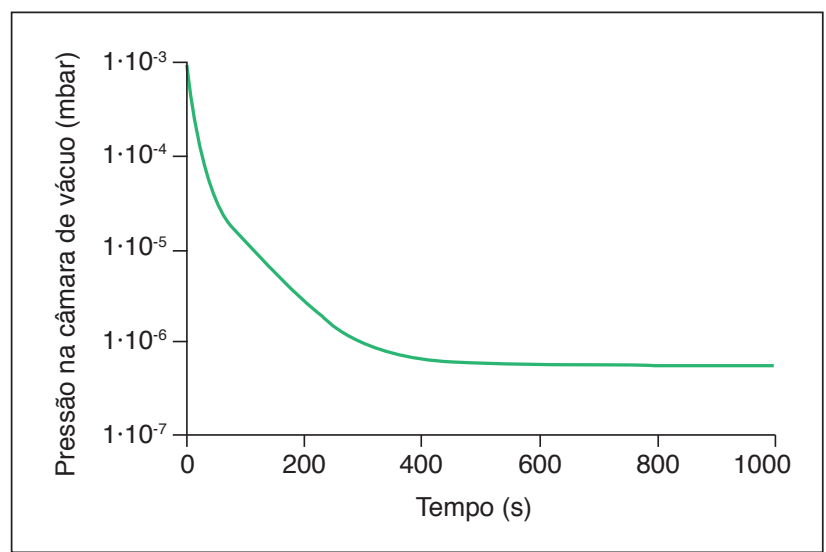

Figura 9: Função temporal da pressão na câmara de vácuo principal com a presença de vazamento virtual. Em 400 segundos, a pressão na câmara de vácuo atinge seu valor final. Nesse caso, o vazamento virtual não é tão danoso ao sistema de vácuo. 


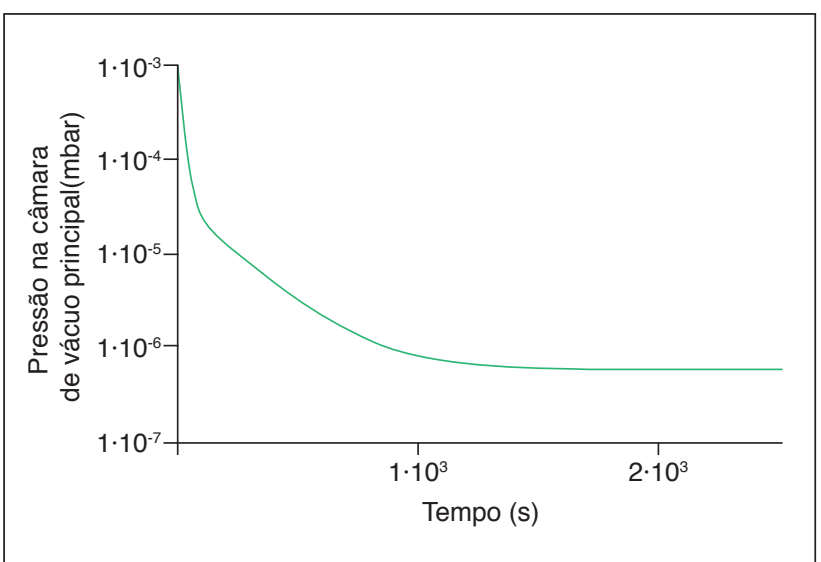

Figura 10: Função temporal da pressão na câmara de vácuo principal com a presença de vazamento virtual. Nesse caso, a pressão na câmara de vácuo demora em torno de 1.200 segundos para alcançar o valor de pressão final. Assim, o vazamento virtual compromete o tempo de espera para realização do processo em vácuo.

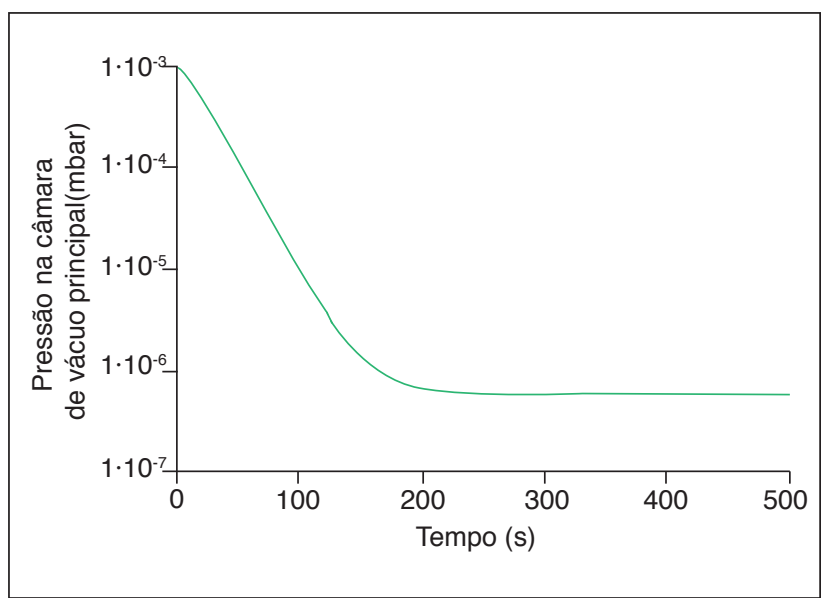

Figura 11: Função temporal da pressão na câmara de vácuo principal com a presença de vazamento virtual. O vazamento virtual não tem efeito sobre o sistema de vácuo. A pressão final na câmara de vácuo atinge o valor estabelecido.

\section{DISCUSSÃO E CONCLUSÃO}

Finalizando este, cabe novamente enfatizar que o vazamento virtual pode comprometer seriamente o bom desempenho de um sistema de vácuo. Nesse sentido, devemos ter especial atenção no projeto, construção e instalação das partes componentes dos sistemas de vácuo, a fim de evitar qualquer formação que neles introduza volumes de gases aprisionados (bolsões de gases nos sistemas de vácuo). Assim, a modelagem do vazamento virtual e a medição da pressão em função do tempo na câmara de vácuo principal possibilitam ou auxiliam a identificação dessa fonte de gases nos sistemas de vácuo. Igualmente, podemos também fazer uso do conhecimento do comportamento do vazamento virtual para estudar o grau de estanqueidade de volumes de sistemas de vácuo selados.

Acrescentando, podemos construir uma série de situações encontradas na tecnologia do vácuo, sofisticações da modelagem do vazamento virtual. Assim, podemos ter a câmara de vácuo principal ligada a duas pequenas câmaras de vácuo pequenas, sendo essas conectadas entre si por um pequeno tubo. Ou ainda, três câmaras de vácuo pequenas conectadas entre si por pequenos tubos. Essas situações não são comumente encontradas nos sistemas de vácuo, mas há aplicações em vácuo que usam as situações descritas. Podemos usar, ainda, a modelagem do vazamento virtual para tratar sistemas de vácuo que apresentem estrutura de bombeamento diferencial. Nesses casos, podemos, em conjunto com a análise específica para bombeamento diferencial, construir uma análise baseada na modelagem do vazamento virtual. Enfim, esse tipo de procedimento está sempre presente na modelagem de sistemas físicos, ou seja, na adaptação de modelos já realizados a outras situações.

\section{TRABALHOS FUTUROS}

Com o propósito de aprimorar a modelagem do vazamento virtual, podemos considerar as seguintes sofisticações:

- Levar em conta a desgaseificação tanto da câmara de vácuo principal como da câmara de vazamento virtual dependente do tempo e da temperatura;

- Considerar que na variação da pressão na câmara do vazamento virtual ocorre o chamado efeito de blocagem, ou seja, o gás está em escoamento blocado para a câmara de vácuo principal;

- Considerar que a condutância da passagem câmara do vazamento virtual para a câmara de vácuo principal é função da pressão (tipo de escoamento do gás).

Assim, a construção de modelos mais sofisticados trará maior compreensão sobre o comportamento de sistemas de vácuo em geral. Teremos, ainda, que desenvolver um substancial ferramental, tanto teórico como de cálculo numéricocomputacional, para resolver os problemas matemáticos estabelecidos.

\section{REFERÊNCIAS}

1. Degasperi FT. Cadernos de atividades - Notas particulares. Instituto de Física da Universidade de São Paulo; 1990, 1991 e 1993.

2. Degasperi FT. Dedução da equação diferencial para o vazamento virtual e sua solução - Objetivo didático. XI Congresso Brasileiro de Aplicações de Vácuo na Indústria e na Ciência; 1990; São Paulo, Brasil.

3. Degasperi FT. Modelagem e análise detalhadas de sistemas de vácuo [dissertação]. Campinas (SP): Universidade Estadual de Campinas, Faculdade de Engenharia Elétrica e de Computação; 2002.

4. Degasperi FT. Contribuições para análise, cálculo e modelagem de sistemas de vácuo [tese]. Campinas (SP): Universidade Estadual de Campinas, Faculdade de Engenharia Elétrica e de Computação; 2006. 
5. Degasperi FT. Modelagem para o vazamento virtual. Seminário Centro Tecnológico para a Informática; 1990; Campinas, Brasil.

6. Harris NS. Modern Vacuum Practice. 3a ed. London: BOC Edwards; 2005.

7. Degasperi FT. Dedução da equação fundamental para o processo de bombeamento na tecnologia do vácuo. Objetivo didático. XII Congresso Brasileiro de Aplicações de Vácuo na Ciência e na Indústria; 1991; Florianópolis, Brasil. Seminário.

8. Ueta K, Watari K. Equações diferenciais ordinárias - Apostilas da disciplina de métodos da física teórica. Instituto de Física da Universidade de São Paulo; 1976. 